\title{
Bayesian estimation for Rayleigh distribution based on ranked set sampling
}

\author{
Saeid Tahmasebi ${ }^{1}$, Elham Haji Hosseini ${ }^{1}$, Ali Akbar Jafari ${ }^{2}$ \\ ${ }^{1}$ Department of Statistics, Persian Gulf University, Bushehr, Iran \\ ${ }^{2}$ Department of Statistics, Yazd University, Yazd, Iran
}

Received: 8 December 2016, Accepted: 17 April 2017

Published online: 8 November 2017.

\begin{abstract}
The Rayleigh distribution is an important model in applications such as noise theory, height of the sea waves and wave length. In this paper, we provide Bayesian estimation for a parameter of the Rayleigh distribution based on simple random sample (SRS) and ranked set sampling (RSS) and maximum ranked set sampling procedure with unequal samples (MRSSU) in two cases, one cycle and m-cycle. We also obtain the Bayes estimators by using square-root inverted-gamma and Jeffreys prior under squared error loss function and general entropy loss function and LINEX function. Finally, we compute the bias and mean squared error of an estimator under squared error and compare its with the corresponding RSS and MRSSU through Monte Carlo simulations.
\end{abstract}

Keywords: Ranked set sampling, Rayleigh distribution, Conjugate prior, Jeffreys prior, Bayes estimator.

\section{Introduction}

McIntyre (1952) first proposed ranked set sampling (RSS) to estimate the mean pasture yields and indicated that RSS is a more efficient sampling method in comparison with simple random sampling(SRS) in terms of the population mean estimation. The one-cycle RSS involves an initial ranking of $n$ samples of size $n$ as follows:

$$
\begin{aligned}
& 1: X_{(1: n) 1} X_{(2: n) 1} \cdots X_{(n: n) 1} \rightarrow Y_{1}=X_{(1: n) 1} \\
& 2: \overline{X_{(1: n) 2}} \underline{X_{(2: n) 2}} \cdots X_{(n: n) 2} \rightarrow Y_{2}=X_{(2: n) 2} \\
& n: X_{(1: n) n} X_{(2: n) n} \cdots \underline{X_{(n: n) n}} \rightarrow Y_{n}=X_{(n: n) n}
\end{aligned}
$$

where $X_{(i: n) j}$ denotes the $i$ th order statistic from the $j t h$ SRS of size $n$. The resulting sample is called one-cycle RSS of size $n$ and denoted by $\underline{Y}=\left(Y_{1}, Y_{2}, \ldots, Y_{n}\right)$. Under the assumption of perfect judgment ranking, $Y_{i}$ has the same distribution as $X_{(i) i}$ which is the $i$ th order statistic in a set of size $n$ obtained from the $i$ th sample with pdf

$$
f_{(i)}(x)=\frac{n !}{(i-1) !(n-i) !} f(x)[F(x)]^{i-1}[1-F(x)]^{n-i} .
$$

The cycle may be repeated $m$ times until $\mathrm{nm}$ units have been quantified. Now, we assume that we have a RSS from a Rayleigh distribution with probability density function (pdf) and the cumulative distribution function (cdf) are given as

$$
f(x ; \sigma)=\frac{x}{\sigma^{2}} \exp \left(\frac{-x^{2}}{2 \sigma^{2}}\right), x>0, \sigma>0,
$$


and

$$
F(x ; \sigma)=1-\exp \left(\frac{-x^{2}}{2 \sigma^{2}}\right),
$$

respectively. To derive the Bayesian estimation of $\sigma$, it is most common to use square error loss(SEL) function, defined as

$$
L_{1}(\hat{\sigma}, \sigma)=(\hat{\sigma}-\sigma)^{2}
$$

where $\hat{\sigma}$ is the estimate of $\sigma$. It may be noted here that (3) defines a symmetric loss function which may be suitable for estimation of $\sigma$. In many practical situations it is more realistic to express the loss in terms of ratio $\frac{\hat{\sigma}}{\sigma}$. In this case, Calabria and Pulcini (1996) proposed a loss function, the general entropy loss(GEL) function of the form:

$$
L_{2}(\hat{\sigma}, \sigma) \propto\left[\left(\frac{\hat{\sigma}}{\sigma}\right)^{p}-p \ln \left(\frac{\hat{\sigma}}{\sigma}\right)-1\right], p \neq 0
$$

where $p$ is the loss parameter which reflects the departure from symmetry. The loss parameter $p$ allows different shapes of this loss function. The LINEX loss function is one of the most popular asymmetric loss function. It was first introduced by Varian (1975) and was extensively discussed by Zellner (1986). The LINEX loss function is given by

$$
L_{3}(\hat{\sigma}, \sigma) \propto \exp (c(\hat{\sigma}-\sigma))-c(\hat{\sigma}-\sigma)-1, c \neq 0
$$

The sign and magnitude of $c$ represents the direction and degree of symmetry, respectively. For $c$ close to zero, the LINEX loss is approximately squared error loss and therefore almost symmetric. In this paper, we also consider the square-root inverted-gamma prior for $\sigma$ which has the form

$$
\pi(\sigma \mid a, b)=\frac{a^{b}}{\Gamma(b) 2^{b-1}}(\sigma)^{-2 b-1} e^{-\frac{a}{2 \sigma^{2}}}
$$

where $a>0$ and $b>0$. When $a=b=0$, it is the non-informative Jefferys prior of $\sigma$. The square-root inverted-gamma prior was first proposed by Bernardo and Smith (1994) and has been used earlier by Fernandez (2000), Raqab and Madi (2002), Wu et al. (2006) and Soliman and Al-Aboud (2008). Many authors have used the RSS for Bayesian estimation of some distributions. Al-Saleh and Muttlak (1998) investigated Bayesian estimators of the mean of the exponential distribution. Kim and Arnold (1999) considered Bayesian estimation under generalized RSS. The concept of Bayesian methods along with RSS was studied by Al-Saleh et al.(2000), who found that for exponential distribution with conjugate prior, the RSS Bayes estimator has smaller Bayes risk than SRS Bayes estimator. Al-Saleh and Abu Hawwas(2002) considered characterization of Bayesian estimation under RSS for normal distribution. Sadek et al.(2015) used the asymmetric loss function to derive the Bayesian estimate based on RSS. Dey et al.(2016) provided Bayes estimator of the scale parameter of Rayleigh distribution under the different sampling schemes. The organization of this article is as follows: In Section 2, we present the Bayes estimates of the parameter $\sigma$ based on both SRS and RSS. In Section 3, we develop Bayesian estimation for the parameter $\sigma$ using MRSSU. Finally, in Section 4, we compute the bias and mean squared error of an estimator under squared error and compare its with the corresponding RSS and MRSSU through Monte Carlo simulations.

\section{Bayes estimates}

In this section, we obtain the Bayes estimates of the parameter $\sigma$ based on both SRS and RSS. In each case, we use both conjugate prior and the non-informative prior and extended Jeffreys prior for $\sigma$. Also, we consider the squared error loss function and general entropy loss function and LINEX function to derive the corresponding Bayesian estimates. Throughout the paper, let $\pi(\sigma \mid \underline{x})$ and $\pi(\sigma \mid \underline{y})$ denote the posterior densities of $\sigma$, given $\operatorname{SRS}(\underline{x})$ and RSS $(\underline{y})$, respectively. 


\subsection{Bayes estimation based on SRS}

Let $X_{1}, X_{2}, \ldots, X_{n}$ be iid random variables from a Rayleigh distribution with parameter $\sigma$ in (1), and $\pi(\sigma)$ be the conjugate prior in (6). Then, the posterior density based on SRS can be obtained as

$$
\pi(\sigma \mid \underline{x})=\frac{2^{1-n-b}\left(a+\sum_{i=1}^{n} x_{i}^{2}\right)^{n+b}}{\Gamma(n+b) \sigma^{2 n+2 b+1}} e^{\frac{-\left(a+\sum_{i=1}^{n} x_{i}^{2}\right)}{2 \sigma^{2}}} .
$$

Hence, the Bayesian estimate of $\sigma$ under SEL function is given by

$$
\tilde{\sigma}_{S E L}(\underline{x})=E(\sigma \mid \underline{x})=\frac{\Gamma\left(n+b-\frac{1}{2}\right)}{\Gamma(n+b)} \sqrt{\frac{\sum_{i=1}^{n} x_{i}^{2}+a}{2}} .
$$

Similarly, the Bayesian estimates of $\sigma$ based on GEL function is obtained as

$$
\tilde{\sigma}_{G E L}(\underline{x})=\left[E\left(\sigma^{-p} \mid \underline{x}\right)\right]^{\frac{-1}{p}}=\left[\frac{\Gamma\left(n+b+\frac{p}{2}\right)}{\Gamma(n+b)}\right]^{\frac{-1}{p}} \sqrt{\frac{\sum_{i=1}^{n} x_{i}^{2}+a}{2}} .
$$

For $p=-1$, equation (9) provides the Bayes estimator under SEL for $\sigma$. Also, the Bayes estimator of $\sigma$ under the LINEX loss function is given by

$$
\tilde{\sigma}_{\text {LINEX }}(\underline{x})=-\frac{1}{c} \ln \left(E\left[e^{-c \sigma}\right]\right),
$$

where

$$
\begin{aligned}
E\left[e^{-c \sigma}\right] & =\int_{0}^{+\infty} \frac{2^{1-n-b}\left(a+\sum_{i=1}^{n} x_{i}^{2}\right)^{n+b}}{\Gamma(n+b) \sigma^{2 n+2 b+1}} \exp \left(\frac{-\left(a+\sum_{i=1}^{n} x_{i}^{2}\right)}{2 \sigma^{2}}-c \sigma\right) d \sigma \\
& =\int_{0}^{+\infty} \frac{2^{1-n-b}\left(a+\sum_{i=1}^{n} x_{i}^{2}\right)^{n+b}}{\Gamma(n+b) \sigma^{2 n+2 b+1}} e^{\frac{-\left(a+\sum_{i=1}^{n} x_{i}^{2}\right)}{2 \sigma^{2}}} \times\left[\sum_{k=0}^{\infty} \frac{(-c \sigma)^{k}}{k !}\right] d \sigma \\
& =\sum_{k=0}^{\infty} \frac{(-c)^{k}}{k !} E\left(\sigma^{k} \mid \underline{x}\right)=\sum_{k=0}^{\infty} \frac{(-c)^{k}}{k !}\left[\frac{\Gamma\left(n+b-\frac{k}{2}\right)}{\Gamma(n+b)}\right]\left(\frac{\sum_{i=1}^{n} x_{i}^{2}+a}{2}\right)^{\frac{k}{2}} .
\end{aligned}
$$

\subsection{Bayes estimate based on RSS}

Let $Y_{1}, Y_{2}, \ldots, Y_{n}$ be a one-cycle RSS from the Rayleigh distribution in (1), and the prior density of $\sigma$ be as in (6). The density of the jth order statistic $Y_{j}$ is known to be

$$
\begin{aligned}
g\left(y_{j} \mid \sigma\right) & =j\left(\begin{array}{c}
n \\
j
\end{array}\right) f\left(y_{j} \mid \sigma\right)\left[F\left(y_{j} \mid \sigma\right)\right]^{j-1}\left[1-F\left(y_{j} \mid \sigma\right)\right]^{n-j} \\
& =j\left(\begin{array}{l}
n \\
j
\end{array}\right) \frac{y_{j}}{\sigma^{2}} \exp \left(\frac{-y_{j}^{2}}{2 \sigma^{2}}\right)\left[1-\exp \left(\frac{-y_{j}^{2}}{2 \sigma^{2}}\right)\right]^{j-1}\left[\exp \left(\frac{-y_{j}^{2}}{2 \sigma^{2}}\right)\right]^{n-j} \\
& =\sum_{k=0}^{j-1} j\left(\begin{array}{c}
n \\
j
\end{array}\right)\left(\begin{array}{c}
j-1 \\
k
\end{array}\right)(-1)^{k} \frac{y_{j}}{\sigma^{2}}\left[\exp \left(\frac{-y_{j}^{2}}{2 \sigma^{2}}\right)\right]^{n-j+k+1} \\
& =\sum_{k=0}^{j-1} t_{k}(j) h_{k}\left(y_{j} \mid \sigma\right)
\end{aligned}
$$


where $t_{k}(j)=j\left(\begin{array}{c}n \\ j\end{array}\right)\left(\begin{array}{c}j-1 \\ k\end{array}\right)(-1)^{k}$ and $h_{k}\left(y_{j} \mid \sigma\right)=\frac{y_{j}}{\sigma^{2}}\left[\exp \left(\frac{-y_{j}^{2}}{2 \sigma^{2}}\right)\right]^{n-j+k+1}$. Then, the joint density of the RSS in this case is given by

$$
\begin{aligned}
g(\underline{y} \mid \sigma) & =\prod_{j=1}^{n} g\left(y_{j} \mid \sigma\right)=\prod_{j=1}^{n} \sum_{k=0}^{j-1} t_{k}(j) h_{k}\left(y_{j} \mid \sigma\right) \\
& =\sum_{i_{1}=0}^{0} \sum_{i_{2}=0}^{1} \ldots \sum_{i_{n}=0}^{n-1}\left[\prod_{j=1}^{n} y_{j} t_{i_{j}}(j)\right] \frac{1}{\sigma^{2 n}} \exp \left(\frac{-\sum_{j=1}^{n} y_{j}^{2}\left(n-j+i_{j}+1\right)}{2 \sigma^{2}}\right)
\end{aligned}
$$

Hence, the posterior density can be derived as

$$
g(\sigma \mid \underline{y})=\frac{\sum_{i_{1}=0}^{0} \sum_{i_{2}=0}^{1} \ldots \sum_{i_{n}=0}^{n-1}\left[\prod_{j=1}^{n} t_{i_{j}}(j)\right] \frac{1}{\sigma^{2 n+2 b+1}} \exp \left(\frac{-\sum_{j=1}^{n} y_{j}^{2}\left(n-j+i_{j}+1\right)-a}{2 \sigma^{2}}\right)}{\sum_{i_{1}=0}^{0} \sum_{i_{2}=0}^{1} \ldots \sum_{i_{n}=0}^{n-1}\left[\prod_{j=1}^{n} t_{i_{j}}(j)\right]\left[\sum_{j=1}^{n} y_{j}^{2}\left(n-j+i_{j}+1\right)+a\right]^{-n-b} \Gamma(n+b) 2^{n+b-1}},
$$

and the Bayesian estimate of $\sigma$ based on the SEL function is obtained from (11) as

$$
\tilde{\sigma}_{S E L}(\underline{y})=\frac{\sum_{i_{1}=0}^{0} \sum_{i_{2}=0}^{1} \ldots \sum_{i_{n}=0}^{n-1}\left[\prod_{j=1}^{n} t_{i_{j}}(j)\right]\left[\sum_{j=1}^{n} y_{j}^{2}\left(n-j+i_{j}+1\right)+a\right]^{-n-b+\frac{1}{2}} \Gamma\left(n+b-\frac{1}{2}\right) 2^{n+b-\frac{3}{2}}}{\sum_{i_{1}=0}^{0} \sum_{i_{2}=0}^{1} \ldots \sum_{i_{n}=0}^{n-1}\left[\prod_{j=1}^{n} t_{i_{j}}(j)\right]\left[\sum_{j=1}^{n} y_{j}^{2}\left(n-j+i_{j}+1\right)+a\right]^{-n-b} \Gamma(n+b) 2^{n+b-1}} .
$$

Also, the Bayesian estimate of $\sigma$ under GEL function is obtained from (11) as

$$
\tilde{\sigma}_{G E L}(\underline{y})=\left[\frac{\Gamma\left(\frac{2 n+2 b+p}{2}\right)}{\Gamma(n+b)}\right]^{\frac{-1}{p}} \times \frac{\sum_{i_{1}=0}^{0} \sum_{i_{2}=0}^{1} \ldots \sum_{i_{n}=0}^{n-1}\left[\prod_{j=1}^{n} t_{i_{j}}(j)\right]\left[\sum_{j=1}^{n} y_{j}^{2}\left(n-j+i_{j}+1\right)+a\right]^{-n-b+\frac{1}{2}} 2^{n+b-\frac{3}{2}}}{\left.\sum_{i_{1}=0}^{0} \sum_{i_{2}=0}^{1} \ldots \sum_{i_{n}=0}^{n-1}\left[\prod_{j=1}^{n} t_{i_{j}}(j)\right]\left[\sum_{j=1}^{n} y_{j}^{2}\left(n-j+i_{j}+1\right)+a\right)\right]^{-n-b} 2^{n+b-1}} .
$$

The Bayesian estimate of $\sigma$ based on the LINEX loss function is given by

$$
\tilde{\sigma}_{\text {LINEX }}(\underline{y})=-\frac{1}{c} \ln \left(E\left[e^{-c \sigma}\right]\right),
$$

where $E\left[e^{-c \sigma}\right]$ is obtained as follows

$$
E\left[e^{-c \sigma}\right]=\sum_{k=0}^{\infty} \frac{(-c)^{k}}{k !} \frac{\Gamma\left(\frac{2 n+2 b-k}{2}\right)}{\Gamma(n+b)} \times\left[\frac{\sum_{i_{1}=0}^{0} \sum_{i_{2}=0}^{1} \ldots \sum_{i_{n}=0}^{n-1}\left[\prod_{j=1}^{n} t_{i_{j}}(j)\right]\left[\sum_{j=1}^{n} y_{j}^{2}\left(n-j+i_{j}+1\right)+a\right]^{-n-b+\frac{1}{2}} 2^{n+b-\frac{3}{2}}}{\sum_{i_{1}=0}^{0} \sum_{i_{2}=0}^{1} \ldots \sum_{i_{n}=0}^{n-1}\left[\prod_{j=1}^{n} t_{i_{j}}(j)\right]\left[\sum_{j=1}^{n} y_{j}^{2}\left(n-j+i_{j}+1+a\right)\right]^{-n-b} 2^{n+b-1}}\right]^{k} .
$$

\subsection{Bayes estimate based on non-informative Jeffreys prior}

Let $\sigma$ be a non-informative Jefferys prior as

$$
\pi(\sigma) \propto \frac{1}{\sigma} .
$$

Then, we obtain the Bayesian estimates of $\sigma$ in cases SRS and RSS as follows 


\section{SRS}

$$
\tilde{\sigma}_{S E L}(\underline{x})=E(\sigma \mid \underline{x})=\frac{\Gamma\left(\frac{2 n-1}{2}\right)}{\Gamma(n)} \sqrt{\frac{\sum_{i=1}^{n} x_{i}^{2}}{2}},
$$

and

$$
\begin{gathered}
\tilde{\sigma}_{G E L}(\underline{x})=\left[E\left(\sigma^{-p} \mid \underline{x}\right)\right]^{\frac{-1}{p}}=\left[\frac{\Gamma\left(\frac{2 n+p}{2}\right)}{\Gamma(n)}\right]^{\frac{-1}{p}} \sqrt{\frac{\sum_{i=1}^{n} x_{i}^{2}}{2}} \\
\tilde{\sigma}_{\text {LINEX }}(\underline{x})=-\frac{1}{c} \ln \left[\sum_{k=0}^{\infty} \frac{(-c)^{k}}{k !}\left[\frac{\Gamma\left(n-\frac{k}{2}\right)}{\Gamma(n)}\right]\left(\frac{\sum_{i=1}^{n} x_{i}^{2}}{2}\right)^{\frac{k}{2}}\right] .
\end{gathered}
$$

2.RSS

$$
\tilde{\sigma}_{S E L}(\underline{y})=\frac{\sum_{i_{1}=0}^{0} \sum_{i_{2}=0}^{1} \ldots \sum_{i_{n}=0}^{n-1}\left[\prod_{j=1}^{n} t_{i_{j}}(j)\right]\left[\sum_{j=1}^{n} y_{j}^{2}\left(n-j+i_{j}+1\right)\right]^{-n+\frac{1}{2}} \Gamma\left(n-\frac{1}{2}\right) 2^{n-\frac{3}{2}}}{\sum_{i_{1}=0}^{0} \sum_{i_{2}=0}^{1} \ldots \sum_{i_{n}=0}^{n-1}\left[\prod_{j=1}^{n} t_{i_{j}}(j)\right]\left[\sum_{j=1}^{n} y_{j}^{2}\left(n-j+i_{j}+1\right)\right]^{-n} \Gamma(n) 2^{n-1}},
$$

and

$$
\begin{aligned}
& \tilde{\sigma}_{G E L}(\underline{y})=\left[\frac{\Gamma\left(\frac{2 n+p}{2}\right)}{\Gamma(n)}\right]^{\frac{-1}{p}} \times \frac{\sum_{i_{1}=0}^{0} \sum_{i_{2}=0}^{1} \ldots \sum_{i_{n}=0}^{n-1}\left[\prod_{j=1}^{n} t_{i_{j}}(j)\right]\left[\sum_{j=1}^{n} y_{j}^{2}\left(n-j+i_{j}+1\right)\right]^{-n+\frac{1}{2}} 2^{n-\frac{3}{2}}}{\sum_{i_{1}=0}^{0} \sum_{i_{2}=0}^{1} \ldots \sum_{i_{n}=0}^{n-1}\left[\prod_{j=1}^{n} t_{i_{j}}(j)\right]\left[\sum_{j=1}^{n} y_{j}^{2}\left(n-j+i_{j}+1\right)\right]^{-n} 2^{n-1}}, \\
& \tilde{\sigma}_{\text {LINEX }}(\underline{y})=-\frac{1}{c} \ln \left\{\sum_{k=0}^{\infty} \frac{(-c)^{k}}{k !} \frac{\Gamma\left(\frac{2 n-k}{2}\right)}{\Gamma(n)} \times\left[\frac{\sum_{i_{1}=0}^{0} \sum_{i_{2}=0}^{1} \ldots \sum_{i_{n}=0}^{n-1}\left[\prod_{j=1}^{n} t_{i_{j}}(j)\right]\left[\sum_{j=1}^{n} y_{j}^{2}\left(n-j+i_{j}+1\right)\right]^{-n+\frac{1}{2}} 2^{n-\frac{3}{2}}}{\sum_{i_{1}=0}^{0} \sum_{i_{2}=0}^{1} \ldots \sum_{i_{n}=0}^{n-1}\left[\prod_{j=1}^{n} t_{i_{j}}(j)\right]\left[\sum_{j=1}^{n} y_{j}^{2}\left(n-j+i_{j}+1\right)\right]^{-n} 2^{n-1}}\right]^{k}\right\} .
\end{aligned}
$$

\subsection{Bayes estimate based on extended Jeffreys prior}

If we consider the extended Jeffreys prior as

$$
\pi(\sigma) \propto \frac{1}{\sigma^{2 c}},
$$

then, we obtain the Bayesian estimates of $\sigma$ in cases SRS and RSS as follows

\section{SRS}

$$
\tilde{\sigma}_{S E L}(\underline{x})=E(\sigma \mid \underline{x})=\frac{\Gamma(n+c-1)}{\Gamma\left(n+c-\frac{1}{2}\right)} \sqrt{\frac{\sum_{i=1}^{n} x_{i}^{2}}{2}},
$$

and

$$
\tilde{\sigma}_{G E L}(\underline{x})=\left[\frac{\Gamma\left(n+c+\frac{p-1}{2}\right)}{\Gamma\left(n+c-\frac{1}{2}\right)}\right]^{\frac{-1}{p}} \sqrt{\frac{\sum_{i=1}^{n} x_{i}^{2}}{2}},
$$




$$
\tilde{\sigma}_{\text {LINEX }}(\underline{x})=-\frac{1}{c} \ln \left[\sum_{k=0}^{\infty} \frac{(-c)^{k}}{k !}\left[\frac{\Gamma\left(n+c-\frac{k+1}{2}\right)}{\Gamma\left(n+c-\frac{1}{2}\right)}\right]\left(\frac{\sum_{i=1}^{n} x_{i}^{2}}{2}\right)^{\frac{k}{2}}\right] .
$$

Note that by replacing $c=\frac{1}{2}$ in (20), the Bayes estimator is obtained as in (16) corresponding to the Jeffreys prior. Replacing $c=\frac{3}{2}$ in (20), the Bayes estimator becomes the estimator under Hartigans prior (Hartigan (1964)).

\section{RSS}

$$
\tilde{\sigma}_{S E L}(\underline{y})=\frac{\sum_{i_{1}=0}^{0} \sum_{i_{2}=0}^{1} \ldots \sum_{i_{n}=0}^{n-1}\left[\prod_{j=1}^{n} t_{i_{j}}(j)\right]\left[\sum_{j=1}^{n} y_{j}^{2}\left(n-j+i_{j}+1\right)\right]^{-n-c+1} \Gamma(n+c-1) 2^{n+c-2}}{\sum_{i_{1}=0}^{0} \sum_{i_{2}=0}^{1} \ldots \sum_{i_{n}=0}^{n-1}\left[\prod_{j=1}^{n} t_{i_{j}}(j)\right]\left[\sum_{j=1}^{n} y_{j}^{2}\left(n-j+i_{j}+1\right)\right]^{-n-c+\frac{1}{2}} \Gamma\left(n+c-\frac{1}{2}\right) 2^{n+c-\frac{3}{2}}},
$$

and

$$
\begin{gathered}
\tilde{\sigma}_{G E L}(\underline{y})=\left[\frac{\Gamma\left(n+c+\frac{p-1}{2}\right)}{\Gamma\left(n+c-\frac{1}{2}\right)}\right]^{\frac{-1}{p}} \times \frac{\sum_{i_{1}=0}^{0} \sum_{i_{2}=0}^{1} \ldots \sum_{i_{n}=0}^{n-1}\left[\prod_{j=1}^{n} t_{i_{j}}(j)\right]\left[\sum_{j=1}^{n} y_{j}^{2}\left(n-j+i_{j}+1\right)\right]^{-n-c+1} 2^{n+c-2}}{\sum_{i_{1}=0}^{0} \sum_{i_{2}=0}^{1} \ldots \sum_{i_{n}=0}^{n-1}\left[\prod_{j=1}^{n} t_{i_{j}}(j)\right]\left[\sum_{j=1}^{n} y_{j}^{2}\left(n-j+i_{j}+1\right)\right]^{-n-c+\frac{1}{2}} 2^{n+c-\frac{3}{2}}}, \\
\tilde{\sigma}_{\text {LINEX }}(\underline{y})=-\frac{1}{c} \ln \left\{\sum_{k=0}^{\infty} \frac{(-c)^{k} \Gamma\left(n+c-\frac{k+1}{2}\right)}{k ! \Gamma\left(n+c-\frac{1}{2}\right)}\left[\frac{\sum_{i_{1}=0}^{0} \sum_{i_{2}=0}^{1} \ldots \sum_{i_{n}=0}^{n-1}\left[\prod_{j=1}^{n} t_{i_{j}}(j)\right]\left[\sum_{j=1}^{n} y_{j}^{2}\left(n-j+i_{j}+1\right)\right]^{-n-c+1} 2^{n+c-2}}{\sum_{i_{1}=0}^{0} \sum_{i_{2}=0}^{1} \ldots \sum_{i_{n}=0}^{n-1}\left[\prod_{j=1}^{n} t_{i_{j}}(j)\right]\left[\sum_{j=1}^{n} y_{j}^{2}\left(n-j+i_{j}+1\right)\right]^{-n-c+\frac{1}{2}} 2^{n+c-\frac{3}{2}}}\right]\right\} .
\end{gathered}
$$

\subsection{Bayes estimate based on m-cycle RSS}

Let $Y_{j l}, j=1,2, \ldots, n, l=1,2, \ldots, m$ be m-cycle RSS from Rayleigh distribution with parameter $\sigma$ in (1) and the prior density of $\sigma$ be the Jeffreys prior. Then, the joint density function in this case is given by

$$
\begin{aligned}
g\left(\underline{y}^{(m)} \mid \sigma\right) & =\prod_{l=1}^{m} \sum_{i_{1}^{l}=0}^{0} \sum_{i_{2}^{l}=0}^{1} \ldots \sum_{i_{n}^{l}=0}^{n-1}\left[\prod_{j=1}^{n} y_{l j} t_{i_{j}^{l}}(j)\right] \frac{1}{\sigma^{2 n}} \exp \left(\frac{-\sum_{j=1}^{n} y_{l j}^{2}\left(n-j+i_{j}^{l}+1\right)}{2 \sigma^{2}}\right) \\
& =\left[\sum_{i_{1}^{l}=0}^{0} \sum_{i_{2}^{l}=0}^{1} \ldots \sum_{i_{n}^{l}=0}^{n-1}\right]\left[\sum_{i_{1}^{2}=0}^{0} \sum_{i_{2}^{2}=0}^{1} \ldots \sum_{i_{n}^{2}=0}^{n-1}\right] \ldots\left[\sum_{i_{1}^{m}=0}^{0} \sum_{i_{2}^{m}=0}^{1} \ldots \sum_{i_{n}^{m}=0}^{n-1}\right] \prod_{l=1}^{m} \prod_{j=1}^{n} t_{i_{j}^{l}}(j) y_{l j} \sigma^{-2 n m} \exp \left(\frac{-\eta_{i_{j}^{l}}}{2 \sigma^{2}}\right),
\end{aligned}
$$

where $\eta_{i_{j}^{l}}=\sum_{l=1}^{m} \sum_{j=1}^{n} y_{l j}^{2}\left(n-j+i_{j}^{l}+1\right)$.

Hence, the posterior density can be expressed as

$$
g\left(\sigma \mid \underline{y}^{(m)}\right)=\frac{\left[\prod_{l=1}^{m} \sum_{i_{1}^{l}=0}^{0} \sum_{i_{2}^{l}=0}^{1} \ldots \sum_{i_{n}^{l}=0}^{n-1}\right] \frac{W_{i_{j}^{l}}}{\sigma^{2 n m+1}} \exp \left(\frac{-\eta_{i_{j}^{l}}}{2 \sigma^{2}}\right)}{\left[\prod_{l=1}^{m} \sum_{i_{1}^{l}=0}^{0} \sum_{i_{2}^{l}=0}^{1} \ldots \sum_{i_{n}^{l}=0}^{n-1}\right] 2^{n m-1}\left(\eta_{i_{j}^{l}}\right)^{-n m} \Gamma(n m)}
$$


where $W_{i_{j}}=\prod_{l=1}^{m} \prod_{j=1}^{n} t_{i_{j}}(j)$. From (21), the Bayesian estimate of $\sigma$ based on the SEL function is given

$$
\tilde{\sigma}_{S E L}\left(\underline{y}^{(m)}\right)=\frac{\left[\prod_{l=1}^{m} \sum_{i_{1}^{l}=0}^{0} \sum_{i_{2}^{l}=0}^{1} \ldots \sum_{i_{n}^{l}=0}^{n-1}\right] W_{i_{j}^{l}} 2^{n m-\frac{3}{2}}\left(\eta_{i_{j}^{l}}\right)^{-n m+\frac{1}{2}} \Gamma(n m-0.5)}{\left[\prod_{l=1}^{m} \sum_{i_{1}^{l}=0}^{0} \sum_{i_{2}^{l}=0}^{1} \ldots \sum_{i_{n}^{l}=0}^{n-1}\right] W_{i_{j}^{l}} 2^{n m-1}\left(\eta_{i_{j}^{l}}\right)^{-n m} \Gamma(n m)}
$$

while the Bayesian estimates of $\sigma$ based on the GEL and LINEX function are derived as

$$
\tilde{\sigma}_{G E L}\left(\underline{y}^{(m)}\right)=\left[\frac{\Gamma\left(\frac{2 n m+p}{2}\right)}{\Gamma(n m)}\right]^{\frac{-1}{p}} \frac{\left[\prod_{l=1}^{m} \sum_{i_{1}^{l}=0}^{0} \sum_{i_{2}^{l}=0}^{1} \ldots \sum_{i_{n}^{l}=0}^{n-1}\right] W_{i_{j}^{l}} 2^{n m-\frac{3}{2}}\left(\eta_{i_{j}^{l}}\right)^{-n m+\frac{1}{2}}}{\left[\prod_{l=1}^{m} \sum_{i_{1}^{l}=0}^{0} \sum_{i_{2}^{l}=0}^{1} \ldots \sum_{i_{n}^{l}=0}^{n-1}\right] W_{i_{j}^{2}} 2^{n m-1}\left(\eta_{i_{j}^{l}}\right)^{-n m}},
$$

and

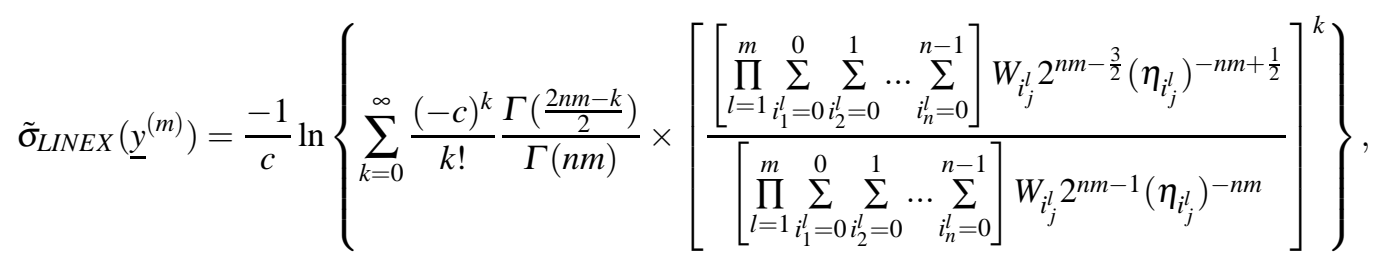

respectively.

\section{Bayesian estimation based on MRSSU}

Biradar and Santosha (2014) proposed maximum ranked set sampling procedure with unequal samples (MRSSU) to estimate the mean of the exponential distribution and indicated that MRSSU is better than those of the estimator based on SRS. The one-cycle MRSSU involves an initial ranking of $n$ samples of size $n$ as follows:

$$
\begin{aligned}
& 1: \underline{X_{(1: 1) 1}} \quad \cdots \quad \rightarrow Z_{1}=X_{(1: 1) 1} \\
& 2: \overline{X_{(1: 2) 2}} \underline{X_{(2: 2) 2} \cdots} \quad \rightarrow Z_{2}=X_{(2: 2) 2} \\
& n: X_{(1: n) n} X_{(2: n) n} \cdots \underline{X_{(n: n) n}} \rightarrow Z_{n}=X_{(n: n) n}
\end{aligned}
$$

The resulting sample is called one-cycle MRSSU of size $n$ and denoted by $\underline{Z}=\left(Z_{1}, Z_{2}, \ldots, Z_{n}\right)$. Under the assumption of perfect judgment ranking, $Z_{i}$ has the same distribution as $X_{(i) i}$ which is the $i$ th order statistic in a set of size $i$ obtained from the $i$ th sample with pdf

$$
f_{(i) i}(z)=i f(z)[F(z)]^{i-1}
$$

The cycle may be repeated $m$ times until $n m$ units have been quantified. Let $Z_{1}, Z_{2}, \ldots, Z_{n}$ be a one-cycle MRSSU from the Rayleigh distribution with parameter $\sigma$ in (1), and the prior density of $\sigma$ be as in (6). The density of the jth order 
statistic(maximum) of an SRS of size $j$ from (1), i.e., $Z_{j}$ is

$$
\begin{aligned}
g\left(z_{j} \mid \sigma\right) & =j f\left(z_{j} \mid \sigma\right)\left[F\left(z_{j} \mid \sigma\right)\right]^{j-1} \\
& =j \frac{z_{j}}{\sigma^{2}} \exp \left(\frac{-z_{j}^{2}}{2 \sigma^{2}}\right)\left[1-\exp \left(\frac{-z_{j}^{2}}{2 \sigma^{2}}\right)\right]^{j-1} \\
& =\sum_{k=0}^{j-1} j\left(\begin{array}{c}
j-1 \\
k
\end{array}\right)(-1)^{k} \frac{z_{j}}{\sigma^{2}}\left[\exp \left(\frac{-z_{j}^{2}}{2 \sigma^{2}}\right)\right]^{k+1} \\
& =\sum_{k=0}^{j-1} q_{k}(j) f_{k}\left(z_{j} \mid \sigma\right),
\end{aligned}
$$

where $q_{k}(j)=j\left(\begin{array}{c}j-1 \\ k\end{array}\right)(-1)^{k}$ and $f_{k}\left(z_{j} \mid \sigma\right)=\frac{z_{j}}{\sigma^{2}}\left[\exp \left(\frac{-z_{j}^{2}}{2 \sigma^{2}}\right)\right]^{k+1}$. Then, the joint density of the MRSSU in this case is given by

$$
\begin{aligned}
g(\underline{z} \mid \sigma) & =\prod_{j=1}^{n} g\left(z_{j} \mid \sigma\right)=\prod_{j=1}^{n} \sum_{k=0}^{j-1} q_{k}(j) f_{k}\left(z_{j} \mid \sigma\right) \\
& =\sum_{i_{1}=0}^{0} \sum_{i_{2}=0}^{1} \ldots \sum_{i_{n}=0}^{n-1}\left[\prod_{j=1}^{n} z_{j} q_{i_{j}}(j)\right] \frac{1}{\sigma^{2 n}} \exp \left(\frac{-\sum_{j=1}^{n} z_{j}^{2}\left(i_{j}+1\right)}{2 \sigma^{2}}\right) .
\end{aligned}
$$

Hence, the posterior density can be derived as

$$
g(\sigma \mid \underline{z})=\frac{\sum_{i_{1}=0}^{0} \sum_{i_{2}=0}^{1} \ldots \sum_{i_{n}=0}^{n-1}\left[\prod_{j=1}^{n} q_{i_{j}}(j)\right] \frac{1}{\sigma^{2 n+2 b+1}} \exp \left(\frac{-\sum_{j=1}^{n} z_{j}^{2}\left(i_{j}+1\right)-a}{2 \sigma^{2}}\right)}{\sum_{i_{1}=0}^{0} \sum_{i_{2}=0}^{1} \ldots \sum_{i_{n}=0}^{n-1}\left[\prod_{j=1}^{n} q_{i_{j}}(j)\right]\left[\sum_{j=1}^{n} z_{j}^{2}\left(i_{j}+1\right)+a\right]^{-n-b} \Gamma(n+b) 2^{n+b-1}},
$$

and the Bayesian estimate of $\sigma$ based on the SEL function is obtained from (22) as

$$
\tilde{\sigma}_{S E L}(\underline{z})=\frac{\sum_{i_{1}=0}^{0} \sum_{i_{2}=0}^{1} \ldots \sum_{i_{n}=0}^{n-1}\left[\prod_{j=1}^{n} q_{i_{j}}(j)\right]\left[\sum_{j=1}^{n} z_{j}^{2}\left(i_{j}+1\right)+a\right]^{-n-b+\frac{1}{2}} \Gamma\left(n+b-\frac{1}{2}\right) 2^{n+b-\frac{3}{2}}}{\sum_{i_{1}=0}^{0} \sum_{i_{2}=0}^{1} \ldots \sum_{i_{n}=0}^{n-1}\left[\prod_{j=1}^{n} q_{i_{j}}(j)\right]\left[\sum_{j=1}^{n} z_{j}^{2}\left(i_{j}+1\right)+a\right]^{-n-b} \Gamma(n+b) 2^{n+b-1}} .
$$

Also, the Bayesian estimate of $\sigma$ under GEL function is obtained from (22) as

$$
\tilde{\sigma}_{G E L}(\underline{z})=\left[\frac{\Gamma\left(\frac{2 n+2 b+p}{2}\right)}{\Gamma(n+b)}\right]^{\frac{-1}{p}} \times \frac{\sum_{i_{1}=0}^{0} \sum_{i_{2}=0}^{1} \ldots \sum_{i_{n}=0}^{n-1}\left[\prod_{j=1}^{n} q_{i_{j}}(j)\right]\left[\sum_{j=1}^{n} z_{j}^{2}\left(i_{j}+1\right)+a\right]^{-n-b+\frac{1}{2}} 2^{n+b-\frac{3}{2}}}{\left.\sum_{i_{1}=0}^{0} \sum_{i_{2}=0}^{1} \ldots \sum_{i_{n}=0}^{n-1}\left[\prod_{j=1}^{n} q_{i_{j}}(j)\right]\left[\sum_{j=1}^{n} z_{j}^{2}\left(i_{j}+1\right)+a\right)\right]^{-n-b} 2^{n+b-1}} .
$$

The Bayesian estimate of $\sigma$ based on the LINEX loss function is given by

$$
\tilde{\sigma}_{\text {LINEX }}(\underline{z})=-\frac{1}{c} \ln \left(E\left[e^{-c \sigma}\right]\right),
$$


where $E\left[e^{-c \sigma}\right]$ is obtained as follows:

$$
E\left[e^{-c \sigma}\right]=\sum_{k=0}^{\infty} \frac{(-c)^{k}}{k !} \frac{\Gamma\left(\frac{2 n+2 b-k}{2}\right)}{\Gamma(n+b)} \times\left[\frac{\sum_{i_{1}=0}^{0} \sum_{i_{2}=0}^{1} \ldots \sum_{i_{n}=0}^{n-1}\left[\prod_{j=1}^{n} q_{i_{j}}(j)\right]\left[\sum_{j=1}^{n} z_{j}^{2}\left(i_{j}+1\right)+a\right]^{-n-b+\frac{1}{2}} 2^{n+b-\frac{3}{2}}}{\sum_{i_{1}=0}^{0} \sum_{i_{2}=0}^{1} \ldots \sum_{i_{n}=0}^{n-1}\left[\prod_{j=1}^{n} q_{i_{j}}(j)\right]\left[\sum_{j=1}^{n} z_{j}^{2}\left(i_{j}+1+a\right)\right]^{-n-b} 2^{n+b-1}}\right]^{k}
$$

\section{Numerical results}

We carry out Mont Carlo simulations using the following steps

(1) Generate SRS and RSS and MRSSU samples of size $n=3(1) 6$ from the Rayleigh distribution with $\sigma=\frac{\sqrt{2}}{2}$ when $m=1$.

(2) Calculate the Bayesian estimates under SEL function using the SRS and RSS and MRSSU samples.

(3) Repeat steps 1 and 2 for 1000 runs.

(4) Then calculate the bias and mean square error (MSE) for all estimates.

In Table(1), the values of bias and MSE of $\sigma$ are obtained based on Jefferys prior for $n=3(1) 6$ and $\sigma=\frac{\sqrt{2}}{2}$. From Table(1), we first of all observe that the Bayesian estimates based on MRSSU are considerably less biased than the corresponding Bayesian estimates on RSS and SRS. Also, we observe that the Bayesian estimates based on MRSSU have much smaller MSE than the corresponding Bayesian estimates based on RSS and SRS in all cases considered. In Table (2), we obtained the values of bias and MSE of $\sigma$ based on the square-root inverted-gamma prior when $n=3(1) 6, \sigma=\frac{\sqrt{2}}{2}$ and $a=b=2$. From Table (2), we first note that the MSE of all estimates decrease when $n$ increases. Next, we can see that MSE of Bayesian estimates using MRSSU are smaller than MSE of Bayesian estimates based on RSS and SRS in all cases. Next, we observe that the estimates based on the square-root inverted-gamma prior are less biased than the corresponding values for estimates based on Jefferys non-informative prior.

Table 1: The values of bias and MSE based on Jefferys prior for $n=3(1) 6$ and $\sigma=\frac{\sqrt{2}}{2}$.

\begin{tabular}{||c|c|c|c||c|c|c|c|}
\hline Bias & & & & MSE & & & \\
\hline$n$ & $S R S$ & $R S S$ & $M R S S U$ & & $S R S$ & $R S S$ & $M R S S U$ \\
\hline 3 & 0.0755 & 0.0333 & 0.0266 & & 0.0059 & 0.0018 & 0.0007 \\
4 & 0.0567 & 0.0221 & 0.0104 & & 0.0042 & 0.0005 & 0.0002 \\
5 & 0.0528 & 0.0167 & 0.0153 & & 0.0033 & 0.0004 & 0.0001 \\
6 & 0.0300 & 0.0123 & 0.0073 & & 0.0019 & 0.0002 & 0.00006 \\
\hline
\end{tabular}

Table 2: Bias and MSE of the Bayesian estimate based on SRS and RSS and MRSSU when $a=b=2$ for $n=3(1) 6$ and $\sigma=\frac{\sqrt{2}}{2}$.

\begin{tabular}{||c|c|c|c||c|c|c|c|}
\hline Bias & & & & MSE & & & \\
\hline$n$ & $S R S$ & $R S S$ & $M R S S U$ & & $S R S$ & $R S S$ & $M R S S U$ \\
\hline 3 & 0.0500 & 0.0308 & 0.0279 & & 0.0022 & 0.0009 & 0.0008 \\
4 & 0.0311 & 0.0180 & 0.0170 & & 0.0011 & 0.0004 & 0.0003 \\
5 & 0.0309 & 0.0118 & 0.0108 & & 0.0009 & 0.0002 & 0.0001 \\
6 & 0.0255 & 0.0062 & 0.0047 & & 0.0008 & 0.0001 & 0.00004 \\
\hline
\end{tabular}




\section{Competing interests}

The authors declare that they have no competing interests.

\section{Authors' contributions}

All authors have contributed to all parts of the article. All authors read and approved the final manuscript.

\section{References}

[1] Al-Saleh, M. F. and Abuhawwas, J. Y. (2002). Characterization of ranked set sampling Bayes estimators with application to the normal distribution. Soochow Journal of Mathematics, 28(2), 223-234.

[2] Al-Saleh, M. F., Al-Shrafat, K., and Muttlak, H. (2000). Bayesian estimation using ranked set sampling. Biometrical journal, 42(4), 489-500.

[3] Al-Saleh, M. F. and Muttlak, H. (1998). A note on the estimation of the parameter of the exponential distribution using Bayesian RSS. Pakistan Journal of Statistics, 14, 49-56.

[4] Bernardo, J. and Smith, A. (1994). Bayesian Theory. Wiley, New York.

[5] Biradar, B.S. and Santosha, C.D. (2014).Estimation of the mean of the exponential distribution using maximum ranked set sampling with unequal samples. Open Journal of Statistics, 4, 641-649.

[6] Dey, S., Salehi, M., and Ahmadi, J.(2016). Rayleigh distribution revisited via ranked set sampling. METRON, DOI 10.1007/s40300-016-0099-2.

[7] Calabria, R. and Pulcini, G. (1996). Point estimation under asymmetric loss functions for left-truncated exponential samples. Communications in Statistics-Theory and methods, 25(3), 585-600.

[8] Fernandez, A. J. (2000). Bayesian inference from type II doubly censored Rayleigh data. Statistics \& Probability Letters, 48(4), 393-399.

[9] Hartigan, J. (1964). Invariant prior distributions. The Annals of Mathematical Statistics, 35(2), 836-845.

[10] Helu, A., Abu-Salih, M., and Alkam, O. (2010). Bayes estimation of Weibull distribution parameters using ranked set sampling. Communications in Statistics-Theory and Methods, 39(14), 2533-2551.

[11] Kim, Y. and Arnold, B. C. (1999). Parameter estimation under generalized ranked set sampling. Statistics \& Probability Letters, 42(4), 353-360.

[12] McIntyre, G. A. (1952). A method for unbiased selective sampling, using ranked sets. Australian Journal of Agricultural Research, 3(4), 385-390.

[13] Raqab, M. and Madi, M. (2002). Bayesian prediction of the total time on test using doubly censored Rayleigh data. Journal of Statistical Computation and Simulation, 72(10), 781-789.

[14] Sadek, A., Sultan, K., and Balakrishnan, N. (2015). Bayesian estimation based on ranked set sampling using asymmetric loss function. Bulletin of the Malaysian Mathematical Sciences Society, 38(2), 707-718.

[15] Soliman, A. A. and Al-Aboud, F. M. (2008). Bayesian inference using record values from Rayleigh model with application. European Journal of Operational Research, 185(2), 659-672.

[16] Varian, H. R. (1975). Bayesian approach to real estate assessment, eds. S. E. Fienberg and A. Zellner. Studies in Bayesian Econometrics and Statistics in Honor of Leonard J. Savage, 195-208. North Holland: Amsterdam.

[17] Wu, S.J., Chen, D.H., and Chen, S.T. (2006). Bayesian inference for Rayleigh distribution under progressive censored sample. Applied Stochastic Models in Business and Industry, 22(3), 269-279.

[18] Zellner, A. (1986). Bayesian estimation and prediction using asymmetric loss functions. Journal of the American Statistical Association, 81(394), 446-451 